
\title{
Research S Surate \\ Extracting high-order cosmological information in galaxy surveys with power spectra
}

\section{Yuting Wang}

National Astronomy Observatories, Chinese Academy of Science

Gong-Bo Zhao ( $\square$ gbzhao@nao.cas.cn )

National Astronomy Observatories, Chinese Academy of Sciences https://orcid.org/0000-0003-4726-

6714

\section{Kazuya Koyama}

University of Portsmouth, UK

Will Percival

University of Waterloo

Ryuichi Takahashi

Hirosaki University

\section{Chiaki Hikage}

Kavli Institute for the Physics and Mathematics of the Universe

Hector Gil-Marin

University of Barcelona

ChangHoon Hahn

Princeton University

Ruiyang Zhao

NAOC

Weibing Zhang

NAOC

Xiaoyong Mu

NAOC

Yu Yu

Shanghai Jiaotong University

Hongming Zhu

CITA

Fei Ge

UC Davis

Article 
Keywords:

Posted Date: February 24th, 2022

DOI: https://doi.org/10.21203/rs.3.rs-1389516/v1

License: (c) (1) This work is licensed under a Creative Commons Attribution 4.0 International License. Read Full License 


\section{Extracting high-order cosmological information in galaxy surveys with power spectra}

Yuting Wang ${ }^{1}$, Gong-Bo Zhao ${ }^{1,2}$, Kazuya Koyama ${ }^{3}$, Will J. Percival ${ }^{4,5,6}$, Ryuichi Takahashi ${ }^{7}$, Chiaki Hikage $^{8}$, Héctor Gil-Marín ${ }^{9}$, ChangHoon Hahn ${ }^{10}$, Ruiyang Zhao ${ }^{2,1}$, Weibing Zhang ${ }^{2,1}$, Xiaoyong $\mathrm{Mu}^{2,1}$, Yu Yu${ }^{11}$, Hong-Ming Zhu ${ }^{12}$, Fei Ge ${ }^{13}$

The "reconstruction" method 1 was proposed more than a decade ago to boost the signal of baryonic acoustic oscillations ${ }^{2}$ measured in galaxy redshift surveys, which is one of key probes for dark energy ${ }^{34}$. After moving observed galaxies in galaxy surveys back to their initial position, the reconstructed density field is closer to a linear Gaussian field, with higher-order information moved back into the power spectrum. By combining power spectra measured from the pre- and post-reconstructed galaxy samples, higher-order information beyond the 2-point power spectrum can be efficiently extracted, as power spectra can be measured more quickly than direct measurements of the bispectrum and other higher order functions. This opens a new window to easily use higher-order information when constraining cosmological models.

The science driver for massive galaxy spectroscopic surveys is to extract cosmological information from the clustering of galaxies in the past lightcone. Baryon acoustic oscillations $(\mathrm{BAO})^{2}$, formed in the early Universe due to interactions between photons and baryons under pressure and gravity, yield a special clustering pattern of galaxies around a characteristic comoving scale around 150 Mpc. The increasing size of galaxy redshift surveys over the decade 2000-2010 led ultimately to 
a $5 \sigma$ detection of BAO by the Baryon Oscillation Spectroscopic Survey (BOSS) ${ }^{5}$. This enabled the BAO to be used as an accurate standard ruler to measure the geometry of the Universe and constrain the cosmic expansion history. A compilation of results from the Sloan Digital Sky Survey (SDSS) galaxy survey recently demonstrated the power of this technique ${ }^{6}$.

The BAO feature is generally blurred by the nonlinear evolution of the Universe reducing its strength as a standard ruler, and various reconstruction methods have been developed to sharpen the BAO peak by undoing the nonlinear evolution of the density field. The commonly used Lagrangian reconstruction, for example, linearises the density field by shifting the galaxies using the displacement field ${ }^{1,78}$, while for the Eulerian reconstruction, manipulation is performed at the field level without moving the galaxies ${ }^{9}$.

Although designed to boost the BAO signal originally, the reconstruction method can, in principle, better extract the general cosmological information from the clustering. For example, the redshift space distortions $(\mathrm{RSD})^{10}$, which lead to clustering pattern of galaxies probing the structure growth of the Universe, can also be better constrained using the reconstructed sample ${ }^{11}$.

The standard method for BAO-reconstruction reverses over-densities back to those of the seed perturbations from which they grew. It achieves this by inferring the bulk-flows using the observed galaxy field and then removing these displacements from both the galaxy positions and the map of expected density setting the baseline from which the over-densities are found. The power spectrum of the post-reconstructed sample $\left(P_{\text {post }}\right)$ provides additional information for cosmology compared with the pre-reconstructed sample $\left(P_{\text {pre }}\right)$ because the reconstruction restores 
the linear signal reduced by the non-linear evolution.

The higher order correlation functions such as $B$, the bispectrum, induced by the non-linear evolution are, in turn, reduced. Thus we can extract more cosmological information encoded in the linear density field from $P_{\text {post }}$ than $P_{\text {pre }}$. On the other hand, the non-linear field contains information on small-scale clustering such as galaxy biases, which provide better constraints on cosmological parameters by breaking the degeneracy between them. For the pre-reconstructed sample, this information can be extracted by combining the power spectrum with higher-order statistics. However, the power spectrum and higher order statistics such as the bispectrum are correlated, reducing our ability to estimate cosmological parameters. If we instead consider the post-reconstructed power spectrum, the covariance between the power spectrum and the bispectrum is reduced, and we can extract the information more efficiently. In this work, we show that the same improvement can be achieved by a joint analysis of $P_{\text {pre }}, P_{\text {post }}$ and $P_{\text {cross }}$ (the cross-power spectrum between the pre- and post-reconstructed density fields). Due to the restored linear signal in the reconstructed density field, $P_{\text {post }}$ is decorrelated with $P_{\text {pre }}$ on small scales, which are dominated by the non-linear field. On these scales, the combination of $P_{\text {pre }}, P_{\text {post }}$ and $P_{\text {cross }}$ has a similar ability to extract cosmological information as the combination of $P_{\text {post }}$ or $P_{\text {pre }}$ with the bispectrum because we are able to use the linear information in $P_{\text {post }}$ and higher-order information in $P_{\text {pre }}$ separately.

Let us rewrite the non-linear over-density field as $\delta=R+\Delta$, where $R$ is the density field after reconstruction, which is closer to the linear field. It is then straightforward to express $P_{\text {pre }}, P_{\text {cross }}$ 
in terms of $P_{R R}\left(=P_{\text {post }}\right), P_{\Delta \Delta}$ (the power spectrum of $\Delta$ ) and $P_{\Delta R}$ (the cross-power spectrum between $\Delta$ and $R$ ). Using perturbation theory ${ }^{[12}$, we can show that, at the leading order, $P_{\Delta \Delta}$ contains the integrated contribution from the bispectrum of squeezed-limit triangles while $P_{R \Delta}$ contains the integrated contribution from the trispectrum of folded/squeeze-limit quadrilaterals (those quadrilaterals with zero area and two-by-two sides equal). In this fashion when we add to the post-reconstructed power spectrum the information from $P_{\text {pre }}$ and $P_{\text {cross }}$, we are not doing anything but including part of the high-order signal, and naturally gaining information. Note that in order to match the information obtained by adding these two extra statistics, it is not enough to consider the bispectrum signal of the pre-reconstructed field, but both bispectrum and trispectrum signals. For this reason the information content of $P_{\text {pre }}+B_{\text {pre }}$ is different from that contained by $P_{\text {pre }}+P_{\text {cross }}+$ $P_{\text {post }}$. However, it is important to note that higher-order information that reconstruction brings is only a part from the total contained by the full bispectrum and trispectrum data-vectors. This is why a full analysis using $P+B+T$ will always provide more information. However, such an analysis is not very practical because of the size of the full data-vector and the computational time typically required to measure $B$ and especially $T$ directly. In this paper we show that $P_{\text {pre }}+P_{\text {cross }}+P_{\text {post }}$ is a practical alternative way of compressing much of the data that captures the most relevant information of these higher-order moments for cosmology inference purposes.

To demonstrate, we perform an anisotropic Lagrangian reconstruction (i.e., the RSD effect is kept during the reconstruction process $s^{1}$, see Method for details) on each realisation of the MOLINO galaxy mocks ${ }^{13}$, which is a large suite of realistic galaxy mocks produced from the Quijote simulations $s^{15}$ at $z=0$. We then use this to calculate the derivatives required for a Fisher 
matrix analysis ${ }^{14}$ using the measured multipoles (up to $\ell=4$ ) of $P_{\text {pre }}, P_{\text {post }}$ and $P_{\text {cross }}$ on the parameter set $\Theta \equiv\left\{\Omega_{\mathrm{m}}, \Omega_{\mathrm{b}}, h, n_{s}, \sigma_{8}, M_{\nu}, \mathbf{H}\right\}$ where $\mathbf{H}$ denotes the Halo Occupation Distribution (HOD) parameters, i.e., $\mathbf{H} \equiv\left\{\log M_{\min }, \sigma_{\log M}, \log M_{0}, \alpha, \log M_{1}\right\}$ ? The covariance matrix and the derivatives with respective to $\Theta$ are evaluated numerically, as in the joint power spectrum-bispectrum analysis $^{13}$ (see Method for details).

Panel a in Figure 1 shows the measured power spectra monopole, and we see that $P_{\text {cross }}$ decreases dramatically with scale compared to $P_{\text {pre }}$ and $P_{\text {post }}$. This indicates a decorrelation between $P_{\text {pre }}$ and $P_{\text {post }}$ below quasi-nonlinear scales $\left(k \gtrsim 0.1 h \mathrm{Mpc}^{-1}\right)$, which is largely due to the difference in levels of nonlinearity in $P_{\text {pre }}$ and $P_{\text {post }}$.

From the original data vector $\left\{P_{\text {pre }}, P_{\text {post }}, P_{\text {cross }}\right\}$, we can construct their linear combinations, $P_{\mathrm{R} \Delta}, P_{\Delta \Delta}, P_{\delta \Delta}$ defined as

$$
P_{\mathrm{R} \Delta}=P_{\text {cross }}-P_{\text {post }}, P_{\Delta \Delta}=P_{\text {pre }}+P_{\text {post }}-2 P_{\text {cross }}, P_{\delta \Delta}=P_{\text {pre }}-P_{\text {cross }} .
$$

Panel b in Figure 1 show these power spectra. As discussed above, these power spectra involving $\Delta$ contain the information on part of the high-order statistics such as bispectrum and trispectrum.

The correlation matrix for the power spectrum and bispectrum monopoles (only the correlation with the squeezed-limit of $B_{0}$ is visualised for brevity) is shown in Figure 2. It is seen that $P_{0}^{\text {pre }}$ highly correlates with $B_{0}$, confirming that the bispectrum is induced by nonlinearities. In contrast, $P_{0}^{\text {post }}$ is almost uncorrelated with $B_{0}$, or with $P_{0}^{\text {pre }}$ and $P_{0}^{\text {cross }}$ on nonlinear scales $(e . g$., at $k \gtrsim 0.2 h \mathrm{Mpc}^{-1}$ ). This implies that the information content in $P_{\text {post }}$ combined with $B_{0}$ may 
be similar to that in $P_{\text {post }}$ combined with $P_{\text {pre }}$ and $P_{\text {cross }}$, which is confirmed to be the case by the results from the Fisher analysis presented below.

Contour plots for $\left(\log M_{0}, \sigma_{8}\right)$ derived from different datasets with two choices of $k_{\max }$ (the maximal $k$ for the observables used in the analysis) are shown in Figure 3 (the full contour plots for all parameters are shown in Supplement Figures 3, 4). The smoothing scale is set to be $S=10 h^{-1} \mathrm{Mpc}$ when performing the reconstruction. The degeneracies between parameters using $P_{\text {pre }}, P_{\text {post }}$ and $P_{\text {cross }}$ are generally different, because $P_{\text {pre }}, P_{\text {post }}$ and $P_{\text {cross }}$ differ to a large extent in terms of nonlinearity on small scales. This is easier to see in Supplement Figure 2, in which contours for the same parameters are shown for observables used in several $k$ intervals. The contours derived from $P_{\text {pre }}$ and $P_{\text {post }}$ generally rotate as $k$ increases because of the kick-in of nonlinear effects, which affects $P_{\text {pre }}$ and $P_{\text {post }}$ at different levels on the same scale. This significantly improves the constraint when these power spectra are combined, labelled as $P_{\text {all }}$, which is tighter than that from the traditional joint power spectrum-bispectrum analysis $\left(P_{\text {pre }}+B_{0}\right)$. Interestingly, $P_{\text {all }}$ can even win against $P_{\text {post }}+B_{0}$ in some cases, demonstrating the robustness of this new method. The contour plots with 1D posterior distributions for all parameters with $S=10$ and $20 h^{-1} \mathrm{Mpc}$ and $k_{\max }=0.2$ and $0.5 h \mathrm{Mpc}^{-1}$ are shown in Supplement Figures 3-6, respectively. In all cases, $P_{\text {all }}$ offers competitive constraints on all parameters, even compared to the joint $P_{\text {post }}+B_{0}$ analysis.

To further quantify our results, in Figure 4 we compare the square root of the Fisher matrix element for each parameter, with and without marginalising over others, derived from $P_{\text {all }}$ and 
$P_{\text {pre }}+B_{0}$, respectively, with two choices of $k_{\max }$.

For $k_{\max }=0.2 h \mathrm{Mpc}^{-1}$, we see that the Fisher information for each parameter (panel a: without marginalising over others) in the model for $P_{\text {all }}$ is identical or even greater than that in $P_{\text {pre }}+B_{0}$. In other words, combining all power spectra we can exhaust the information in $P_{\text {pre }}+B_{0}$. After marginalising over other parameters, panel $\mathbf{b}$ shows that the uncertainty on each parameter gets redistributed due to the degeneracy. The ratios for the HOD parameters are all greater than unity especially for $\log M_{0}$ and $\sigma_{\log M}$, demonstrating the power of our new method on constraining HOD parameters. The information content for cosmological parameters in $P_{\text {pre }}+B_{0}$ is well recovered by using $P_{\text {all }}$, although the recovery for $M_{\nu}$ is relatively worse. The overall trend for the case of $k_{\max }=0.5 \mathrm{~h} \mathrm{Mpc}^{-1}$ is similar, although the advantage of using $P_{\text {all }}$ over $P_{\text {pre }}+B_{0}$ gets degraded to some extent. However, $P_{\text {all }}$ is still competitive: it almost fully recovers the information for the HOD parameters in $P_{\text {pre }}+B_{0}$ with or without marginalisation, and largely wins against $P_{\text {pre }}+B_{0}$ after marginalisation. Regarding the cosmological parameters, $P_{\text {all }}$ recovers all information in $P_{\text {pre }}+B_{0}$ before the marginalisation, although the recovery is slightly worse for $M_{\nu}$. After marginalisation when the uncertainties are redistributed, the constraint from $P_{\text {all }}$ is generally worse than $P_{\text {pre }}+B_{0}$, especially for $M_{\nu}$.

The $68 \%$ CL constraints on each parameter expected fitting to different sets of data are shown in Table 1. To quantify the information gain, we evaluate the Figure-of-Merit (FoM) defined as $[\operatorname{det}(F)]^{1 /\left(2 N_{\mathrm{p}}\right)}$, where $F$ denotes the Fisher matrix and $N_{\mathrm{p}}$ is the total number of free parameters. For the ease of comparison, for cases with different $k_{\max }$, we normalise the FoM using the corresponding 
$(\mathrm{FoM})_{P_{\mathrm{pre}}}$. As shown, for $k_{\max }=0.2 h \mathrm{Mpc}^{-1},(\mathrm{FoM})_{P_{\text {all }}}$ is greater than all others, namely, it is larger than $(\mathrm{FoM})_{P_{\mathrm{pre}}}$ and $(\mathrm{FoM})_{P_{\mathrm{post}}}$ by a factor of 2.7 and 1.6, respectively, and it is even greater than $(\mathrm{FoM})_{P_{\mathrm{post}}+B_{0}}$ by $\sim 13 \%$. For $k_{\max }=0.5 h \mathrm{Mpc}^{-1},(\mathrm{FoM})_{P_{\text {all }}}$ is also more informative than $(\mathrm{FoM})_{P_{\mathrm{pre}}}$ and $(\mathrm{FoM})_{P_{\mathrm{post}}}$ by a factor of 2.1 and 1.5 , respectively, and is the same as $(\mathrm{FoM})_{P_{\mathrm{pre}}+B_{0}}$, but is less than $(\mathrm{FoM})_{P_{\mathrm{post}}+B_{0}}$ by $\sim 10 \%$ in this case.

To highlight the constraining power on cosmological parameters, we also list $\mathrm{FoM}_{\mathrm{cos}}$, which is the FoM with all HOD parameters fixed. It shows a similar trend as $\mathrm{FoM}_{\ominus}: P_{\text {all }}$ is the most informative data combination for $k_{\max }=0.2 \mathrm{~h} \mathrm{Mpc}^{-1}$, but it is outnumbered by $P_{\text {pre }}+B_{0}$ and $P_{\text {post }}+B_{0}$ by $13 \%$ and $35 \%$, respectively, for the case of $k_{\max }=0.5 h \mathrm{Mpc}^{-1}$.

In this example, the $k$-binning for $P$ and $B$ are different, namely, $\Delta k(B)=3 k_{f} \sim 1.9 \Delta k(P)$ where $k_{f}$ denotes the fundamental $k$ mode given the box size of the simulation. We have checked that using a much finer $k$-binning for $B$ does not change significantly the constraints ${ }^{17}$. The FoM can only be raised by $\sim 10 \%$ when $\Delta k(B)$ is reduced from $3 k_{f}$ to $k_{f}$, which is largely due to the strong mode-coupling effect in $B$ shown in Figure 2. Such a binning is not practical anyway as, for example, using $\Delta k(B)=k_{f}$ up to $k=0.5 h \mathrm{Mpc}^{-1}$, we end up with more than 50,000 data points to measure for $B_{0}$.

Another concern might be that the MOLINO mock was investigated at $z=0$, where the nonlinear effect are the strongest. At higher redshifts where the density fields are more linear and more Gaussian, we may expect less gain from the new method. This is also the case for the joint $P+$ $B$ analysis, compared to analyses of only $P$. To quantify, we also perform an independent analysis 
on a suite of 4000 high-resolution $N$-body mocks produced at $z=1.02$, and confirm the gain in cosmological information in terms of BAO and RSD parameters (see Supplement information). Therefore, for redshift ranges covered by ongoing and future galaxy surveys (with median $z \sim 1$ ), our method manifests itself as a highly efficient and powerful way of transferring higher-order information back to the 2-point statistics.

As demonstrated, a joint analysis using $P_{\text {pre }}, P_{\text {post }}$ and $P_{\text {cross }}$ is an efficient way to extract high-order information from galaxy catalogues, and in some cases, the compression of information into $P_{\text {all }}$ is more informative even than to $P_{\text {post }}+B_{0}$, which is more computationally expensive. This may be attributed to the fact that considering the full multipole expansion of $P_{\text {all }}$ in terms of Legendre polynomials includes information from high-multipoles of the bispectrum and trispectrum, which are challenging to measure and use in traditional approaches.

Stage-IV redshift surveys including the Dark Energy Spectroscopic Instrument (DESI) ${ }^{18}$, Euclid $^{19}$ and the Prime Focus Spectrograph (PFS) ${ }^{20}$ will release galaxy maps over a wide range of redshift with an exquisite precision. Given that the number density of these galaxies is expected to be sufficiently high to allow for a reconstruction, the novel method presented in this work can be directly applied to yield a much more precise measurement of cosmological parameters, compared to the traditional 2-point, or even the 2-point +3 -point analyses. Since the reconstruction will be performed anyway for most ongoing and forthcoming galaxy surveys to improve the BAO signal, our proposed analysis can be performed at almost no additional computational cost.

Additional work is required to link the cosmological parameters to the observables, especially 
the reconstructed power spectrum ${ }^{12 \sqrt{21}}$ and the cross power spectrum ${ }^{22}$ on (quasi-) nonlinear scales, and this is challenging using traditional approaches, e.g., methods based on perturbation theories. However, new model-free approaches including the simulation-based emulators, will be ideal for implementing our analysis on actual galaxy surveys.

1. Eisenstein, D. J., Seo, H.-j., Sirko, E. \& Spergel, D. Improving Cosmological Distance Measurements by Reconstruction of the Baryon Acoustic Peak. Astrophys. J. 664, 675-679 (2007).

2. Eisenstein, D. J. \& Hu, W. Baryonic Features in the Matter Transfer Function. Astrophys. J. 496, 605-614 (1998).

3. Riess, A. G. et al. Observational Evidence from Supernovae for an Accelerating Universe and a Cosmological Constant. Astron. J. 116, 1009-1038 (1998).

4. Perlmutter, S. et al. Measurements of $\Omega$ and $\Lambda$ from 42 High-Redshift Supernovae. Astrophys. J. 517, 565-586 (1999).

5. Dawson, K. S. et al. The Baryon Oscillation Spectroscopic Survey of SDSS-III. Astron. J. 145, 10 (2013).

6. Alam, S. et al. Completed SDSS-IV extended Baryon Oscillation Spectroscopic Survey: Cosmological implications from two decades of spectroscopic surveys at the Apache Point Observatory. Phys. Rev. D 103, 083533 (2021). 
7. Padmanabhan, $\mathrm{N}$. et al. A 2 per cent distance to $z=0.35$ by reconstructing baryon acoustic oscillations - I. Methods and application to the Sloan Digital Sky Survey. Mon. Not. Roy. Astron. Soc. 427, 2132-2145 (2012). 1202.0090.

8. Burden, A. et al. Efficient reconstruction of linear baryon acoustic oscillations in galaxy surveys. Mon. Not. Roy. Astron. Soc. 445, 3152-3168 (2014).

9. Schmittfull, M., Feng, Y., Beutler, F., Sherwin, B. \& Chu, M. Y. Eulerian BAO reconstructions and N -point statistics. Phys. Rev. D 92, 123522 (2015).

10. Kaiser, N. Clustering in real space and in redshift space. Mon. Not. Roy. Astron. Soc. 227, $1-27$ (1987).

11. Hikage, C., Takahashi, R. \& Koyama, K. Covariance of the redshift-space matter power spectrum after reconstruction. Phys. Rev. D 102, 083514 (2020).

12. Hikage, C., Koyama, K. \& Takahashi, R. Perturbation theory for the redshift-space matter power spectra after reconstruction. Phys. Rev. D 101, 043510 (2020). 1911.06461.

13. Hahn, C. \& Villaescusa-Navarro, F. Constraining $\mathrm{M}_{\nu}$ with the bispectrum. Part II. The information content of the galaxy bispectrum monopole. JCAP 2021, 029 (2021).

14. Tegmark, M., Taylor, A. \& Heavens, A. Karhunen-Loeve eigenvalue problems in cosmology: How should we tackle large data sets? Astrophys. J. 480, 22 (1997).

15. Villaescusa-Navarro, F. et al. The Quijote Simulations. Astrophys. J., Suppl. Ser. 250, 2 (2020). 
16. Zheng, Z., Coil, A. L. \& Zehavi, I. Galaxy Evolution from Halo Occupation Distribution Modeling of DEEP2 and SDSS Galaxy Clustering. Astrophys. J. 667, 760-779 (2007).

17. Yankelevich, V. \& Porciani, C. Cosmological information in the redshift-space bispectrum. Mon. Not. Roy. Astron. Soc. 483, 2078-2099 (2019).

18. DESI Collaboration. The DESI Experiment Part I: Science,Targeting, and Survey Design. ArXiv e-prints (2016). 1611.00036.

19. Laureijs, R. et al. Euclid Definition Study Report (2011). 1110.3193 .

20. Ellis, R. et al. Extragalactic science, cosmology, and Galactic archaeology with the Subaru Prime Focus Spectrograph. Publ. Astron. Soc. Jap. 66, R1 (2014).

21. Chen, S.-F., Vlah, Z. \& White, M. The reconstructed power spectrum in the Zeldovich approximation. JCAP 09, 017 (2019). 1907.00043.

22. Zhang, W. et al. (To appear).

Correspondence Correspondence and requests for materials should be addressed to G. B. Zhao (email: gbzhao@nao.cas.cn), Kazuya Koyama (email: kazuya.koyama@port.ac.uk) or Will Percival (email: will.percival@uwaterloo.ca).

Acknowledgements We thank Florian Beutler, Shi-Fan Chen, Yipeng Jing, Baojiu Li, Levon Pogosian, Uroš Seljak, Shun Saito, Atsushi Taruya, Francisco Villaescusa-Navarro, Martin White, Hans Winther, Hanyu Zhang and Pengjie Zhang for discussions. YW is supported by NSFC Grant 11890691, by the Youth Innovation Promotion Association CAS, and by the Nebula Talents Program of NAOC. GBZ is 
supported by the National Key Basic Research and Development Program of China (No. 2018YFA0404503), NSFC Grants 11925303, 11720101004, and a grant of CAS Interdisciplinary Innovation Team. Research at Perimeter Institute is supported in part by the Government of Canada through the Department of Innovation, Science and Economic Development Canada and by the Province of Ontario through the Ministry of Colleges and Universities. RT is supported by MEXT/JSPS KAKENHI Grant Numbers 20H05855 and 20H04723. Numeric work was performed on the UK Sciama High Performance Computing cluster supported by the ICG, University of Portsmouth.

Author contributions YW contributed to the idea and the development of the pipeline, performed the analysis on the Molino mocks, produced the results and contributed to the draft. GBZ proposed the idea, developed the pipeline, preformed the analysis on the $N$-body mocks and wrote the draft. KK contributed to the idea and pipeline, led the effort on the theoretical interpretation of the result and co-wrote the draft. WJP contributed to the theoretical interpretation and observational implications of this work, and co-wrote the draft. RT and $\mathrm{CH}$ provided the $\mathrm{N}$-body mocks, performed the reconstruction and measured the power spectra. HGM and $\mathrm{CHH}$ contributed to the bispectrum analysis, and to the draft. WZ, RZ and XM contributed to building an EFT-based theoretical model for the cross-power, which helped with interpretation of the result. YY and $\mathrm{HZ}$ provided another set of $N$-body mock for a cross validation, and helped with interpreting the de-correlation effect. FG preformed a test on the eBOSS mocks to confirm the de-correlation effect between $P_{\text {pre }}$ and $P_{\text {post }}$.

Author Information ${ }^{1}$ National Astronomy Observatories, Chinese Academy of Sciences, Beijing, 100101, P.R.China

${ }^{2}$ School of Astronomy and Space Science, University of Chinese Academy of Sciences, Beijing 100049 , P.R.China 
${ }^{3}$ Institute of Cosmology and Gravitation, University of Portsmouth, Dennis Sciama Building, Portsmouth PO1 3FX, United Kingdom

${ }^{4}$ Waterloo Centre for Astrophysics, University of Waterloo, 200 University Ave W, Waterloo, ON, N2L 3G1, Canada

${ }^{5}$ Department of Physics and Astronomy, University of Waterloo, 200 University Ave W, Waterloo, ON, N2L 3G1, Canada

${ }^{6}$ Perimeter Institute for Theoretical Physics, 31 Caroline St. North, Waterloo, ON, N2L 2Y5, Canada

${ }^{7}$ Faculty of Science and Technology, Hirosaki University, 3 Bunkyo-cho, Hirosaki, Aomori 036-8561, Japan

${ }^{8}$ Kavli Institute for the Physics and Mathematics of the Universe (Kavli IPMU, WPI), University of Tokyo, 5-1-5 Kashiwanoha, Kashiwa, Chiba, 277-8583, Japan

${ }^{9}$ ICC, University of Barcelona, IEEC-UB, Martí i Franquès, 1, E-08028 Barcelona, Spain

${ }^{10}$ Department of Astrophysical Sciences, Princeton University, Peyton Hall, Princeton NJ 08544, USA

${ }^{11}$ Department of Astronomy, Shanghai Jiao Tong University, Shanghai, 200240, P.R.China

${ }^{12}$ Canadian Institute for Theoretical Astrophysics, University of Toronto, 60 St. George Street, Toronto, Ontario M5S 3H8, Canada

${ }^{13}$ Department of Physics and Astronomy, University of California, Davis, California 95616, USA

Competing Interests The authors declare that they have no competing financial interests. 


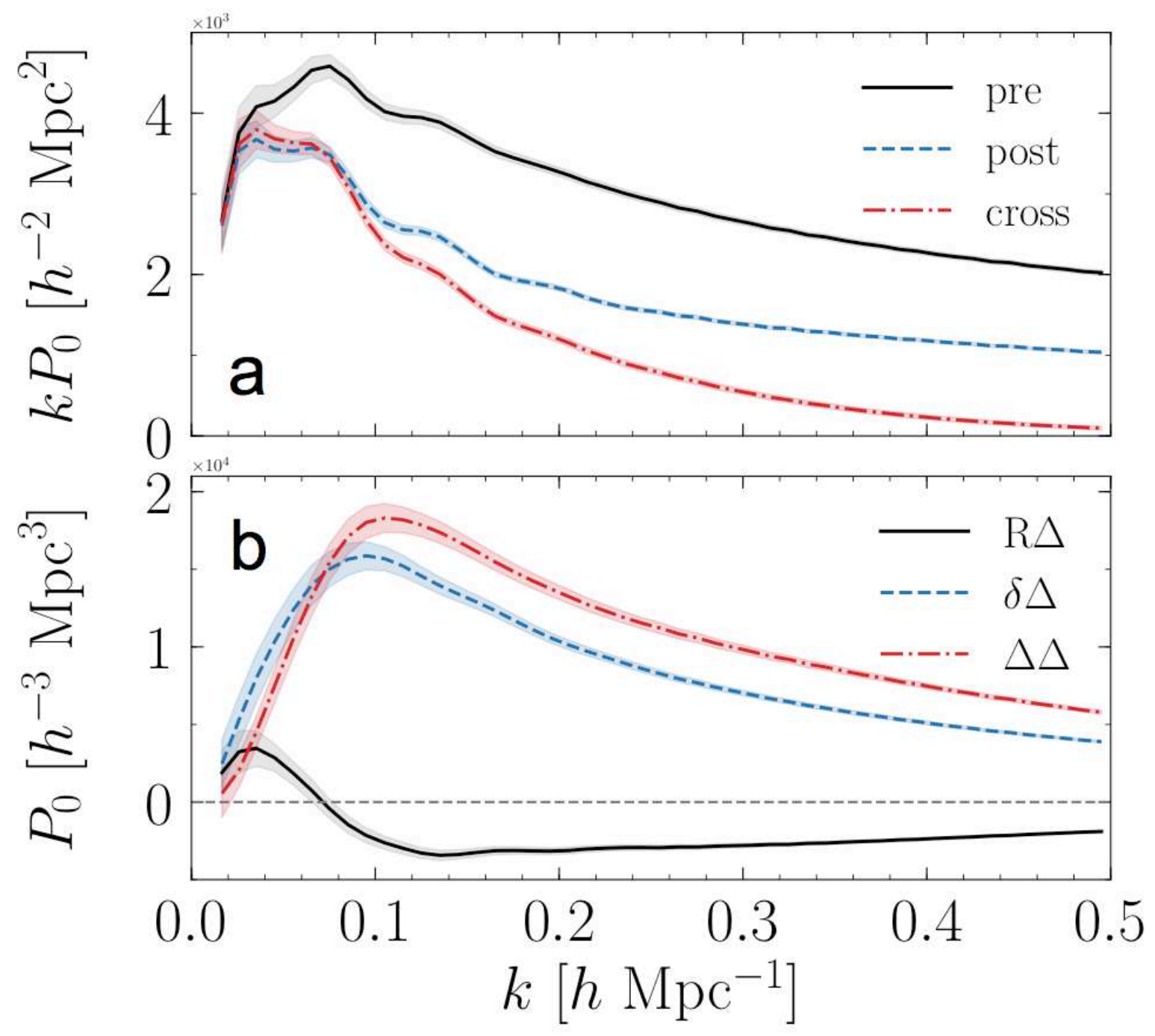

Figure 1. The measured power spectrum monopoles.

Panels a: the monopole (multiplied by $k$ ) of three types of power spectra indicated in the legend, measured from the Molino galaxy mocks; b: the rotated power spectra monopoles as defined in Eq.1. In both panels, the lines in the center denote the mean of the mocks, and the shades represent the $68 \%$ CL uncertainty. 


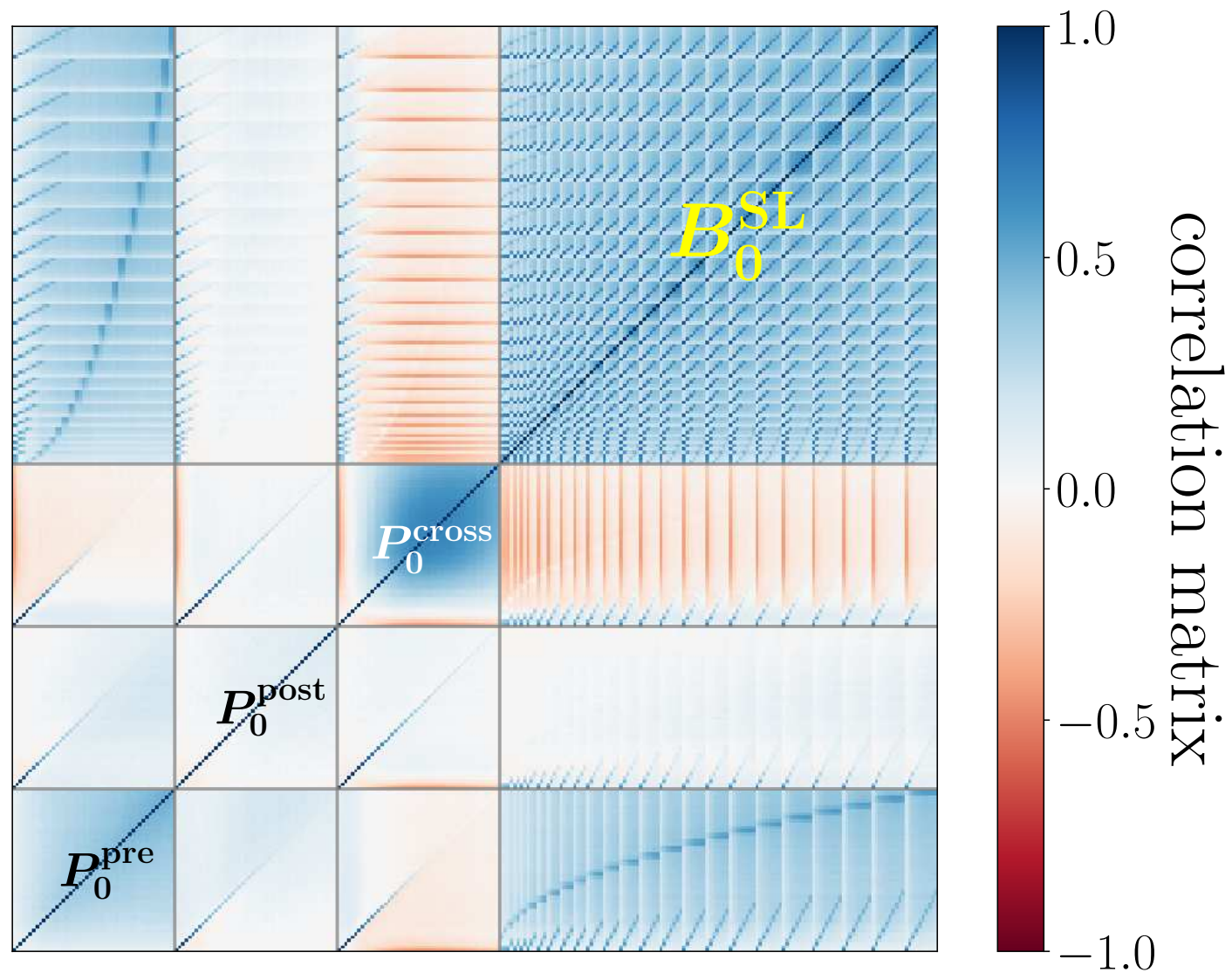

Figure 2. Part of the correlation matrix between the power spectra and bispectrum.

The correlation matrix for the monopoles of three types of power spectra $\left(P_{0}^{\text {pre }}, P_{0}^{\text {post }}, P_{0}^{\text {cross }}\right)$, and of the bispectrum in the squeezed limit $\left(B_{0}^{\mathrm{SL}}\right)$, i.e. $k_{1}=k_{2} \gg k_{3}$, derived from the MoLINo galaxy mocks. The horizontal and vertical lines separate each block for visualisation. For all blocks, the associated $k$ or $k_{1}$ increases from 0.01 to $0.5 \mathrm{~h} \mathrm{Mpc}^{-1}$, from left to right, and from bottom to top. 

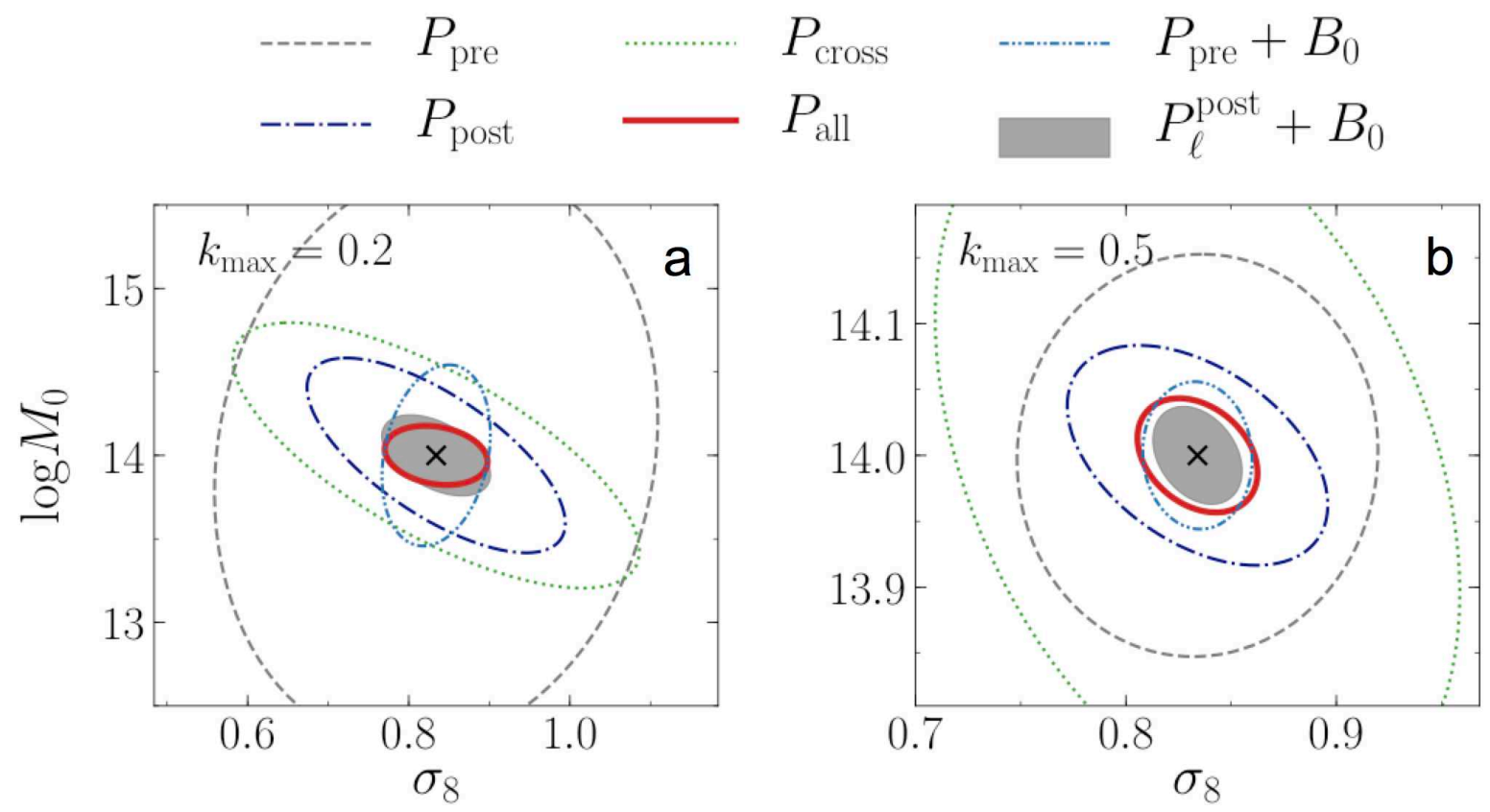

Figure 3. The $68 \%$ CL contour plots on $\sigma_{8}$ and $\log M_{0}$ derived from various data combinations.

Panels a and $\mathbf{b}$ show the constraint with $k_{\max }=0.2$ and $0.5 \mathrm{~h} \mathrm{Mpc}^{-1}$, respectively. 


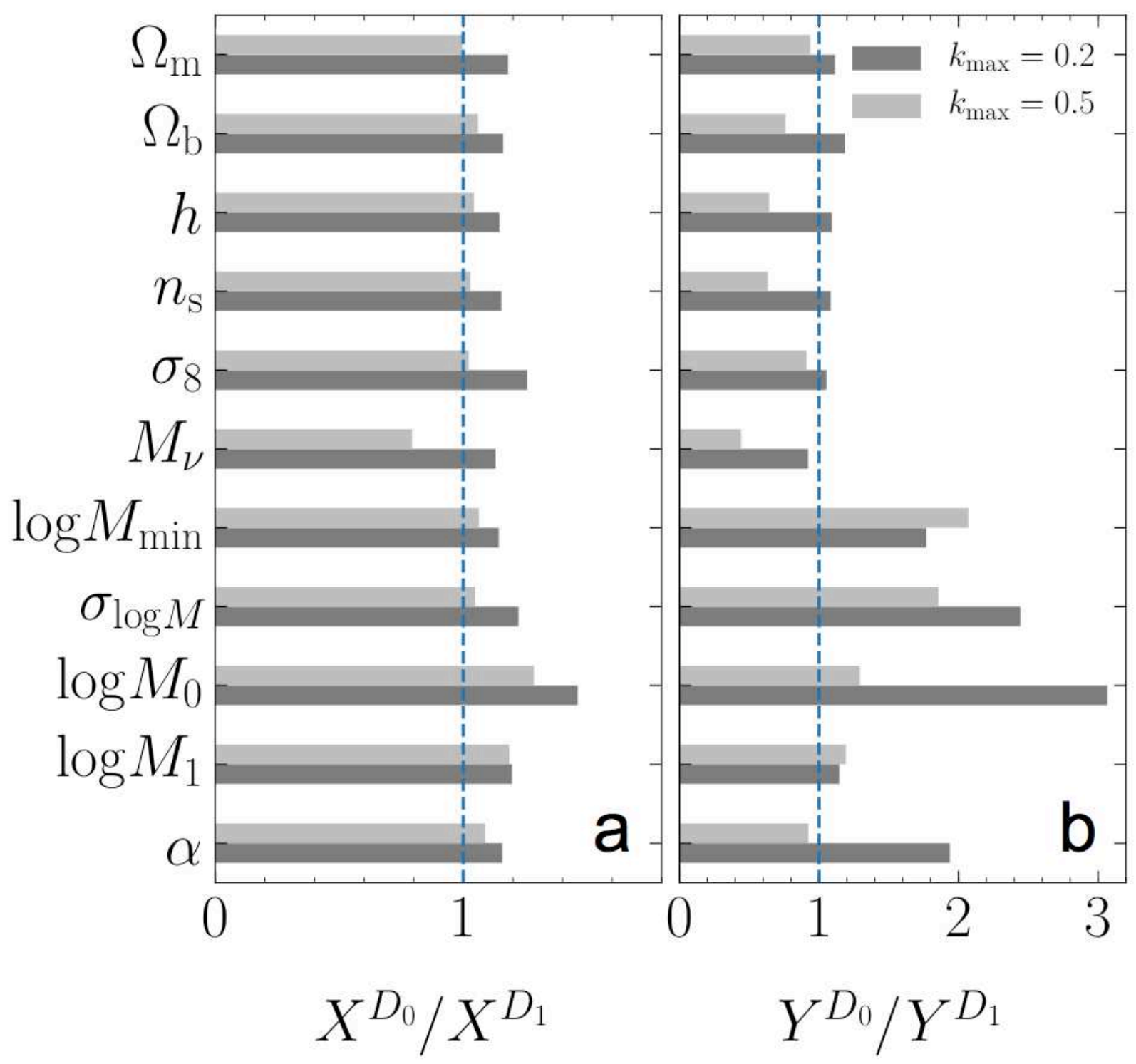

Figure 4. The Figure of Merit (FoM) of each individual parameter derived from all power spectrum combined, rescaled by those derived from $P_{\text {pre }}+B_{0}$.

The quantities $X$ and $Y$ are defined as the FoM of each individual parameter with or without all other parameters fixed. Specifically, $X \equiv \sqrt{F_{i i}}$ and $Y \equiv 1 / \sqrt{C_{i i}}$ where $F$ is the Fisher matrix and $C \equiv F^{-1} . D_{0}$ and $D_{1}$ denote $P_{\text {all }}$ and $P_{\text {pre }}+B_{0}$ respectively. The upper light gray and lower 
dark gray bars show the cases with $k=0.2$ and $0.5 h \mathrm{Mpc}^{-1}$, respectively. The vertical dashed lines shows a full recovery of information from dataset $P_{\text {pre }}+B_{0}$. The smoothing scale is set to be $10 h^{-1} \mathrm{Mpc}$ when performing the reconstruction. 


\begin{tabular}{|c|c|c|c|c|c|c|c|c|c|c|}
\hline & \multicolumn{5}{|c|}{$k_{\max }=0.2 h \mathrm{Mpc}^{-1}$} & \multicolumn{5}{|c|}{$k_{\max }=0.5 h \mathrm{Mpc}^{-1}$} \\
\hline & $P_{\text {pre }}$ & $P_{\text {post }}$ & $P_{\text {all }}$ & $P_{\text {pre }}+B_{0}$ & $P_{\text {post }}+B_{0}$ & $P_{\text {pre }}$ & $P_{\text {post }}$ & $P_{\text {all }}$ & $P_{\text {pre }}+B_{0}$ & $P_{\text {post }}+B_{0}$ \\
\hline$\Omega_{\mathrm{m}}$ & 0.054 & 0.042 & 0.027 & 0.030 & 0.027 & 0.029 & 0.026 & 0.015 & 0.019 & 0.012 \\
\hline$\Omega_{\mathrm{b}}$ & 0.025 & 0.018 & 0.011 & 0.013 & 0.011 & 0.014 & 0.010 & 0.007 & 0.007 & 0.004 \\
\hline$h$ & 0.329 & 0.235 & 0.141 & 0.154 & 0.142 & 0.155 & 0.132 & 0.075 & 0.068 & 0.043 \\
\hline$n_{\mathrm{s}}$ & 0.395 & 0.267 & 0.152 & 0.165 & 0.154 & 0.171 & 0.158 & 0.077 & 0.068 & 0.047 \\
\hline$\sigma_{8}$ & 0.183 & 0.107 & 0.043 & 0.045 & 0.045 & 0.057 & 0.041 & 0.019 & 0.022 & 0.014 \\
\hline$M_{\nu}[\mathrm{eV}]$ & 0.865 & 0.655 & 0.307 & 0.283 & 0.274 & 0.355 & 0.283 & 0.160 & 0.102 & 0.070 \\
\hline $\mathrm{FoM}_{\mathrm{cos}}$ & 1.0 & 1.6 & 2.5 & 2.0 & 2.4 & 1.0 & 1.6 & 2.3 & 2.6 & 3.1 \\
\hline $\log M_{\min }$ & 0.678 & 0.455 & 0.143 & 0.254 & 0.267 & 0.149 & 0.081 & 0.028 & 0.075 & 0.047 \\
\hline$\sigma_{\log M}$ & 1.333 & 0.848 & 0.250 & 0.610 & 0.545 & 0.464 & 0.238 & 0.078 & 0.202 & 0.102 \\
\hline $\log M_{0}$ & 1.170 & 0.389 & 0.118 & 0.362 & 0.162 & 0.102 & 0.056 & 0.029 & 0.040 & 0.025 \\
\hline $\log M_{1}$ & 0.679 & 0.625 & 0.186 & 0.213 & 0.250 & 0.116 & 0.115 & 0.051 & 0.066 & 0.057 \\
\hline$\alpha$ & 0.601 & 0.330 & 0.084 & 0.162 & 0.119 & 0.080 & 0.076 & 0.033 & 0.032 & 0.027 \\
\hline $\mathrm{FoM}_{\Theta}$ & 1.0 & 1.7 & 2.7 & 1.9 & 2.4 & 1.0 & 1.4 & 2.1 & 2.1 & 2.3 \\
\hline
\end{tabular}

Table 1: A quantification of the information content extracted from various observables measured from the Molino mocks. The $68 \% \mathrm{CL}$ uncertainty of parameters derived from different datasets, with two different choices of the maximal wavenumber $k_{\max }$. The last row, $\mathrm{FoM}_{\Theta}$ shows the Figure-of-Merit (FoM), defined as $[\operatorname{det}(F)]^{1 /\left(2 N_{\mathrm{p}}\right)}$, where $F$ denotes the Fisher matrix and $N_{\mathrm{p}}$ is the total number of free parameters. The FoM is normalised by the corresponding $(\mathrm{FoM})_{P_{\mathrm{pre}}}$ for two cases with different $k_{\max }$. The row of $\mathrm{FoM}_{\mathrm{cos}}$ shows the same FoM as defined above, but with all HOD parameters fixed. 


\section{Methods}

\section{The Molino galaxy mocks}

The MoLINO catalogs $\frac{13}{13}$ are suite of publicly available galaxy mock catalogs that were constructed to quantify the total cosmological information content of different galaxy clustering observables using Fisher matrix forecasting $\left.\right|^{1}$. They are constructed from the QUIJOTE suite of $N$-body simulations $s^{15}$ using the halo occupation distribution (HOD) framework. HOD provides a statistical prescription for populating dark matter halos with central and satellite galaxies and has been successful in reproducing a wide range of observed galaxy clustering statistics. In particular, the MOLINO catalogs use the standard ${ }^{\sqrt{16}}$ HOD model, which has five free parameters: $\left\{\log M_{\min }, \sigma_{\log M}, \log M_{0}\right.$, $\left.\alpha, \log M_{1}\right\}$. MoLino includes 15,000 galaxy catalogs that are constructed at a fiducial set of cosmological parameters $\left(\Omega_{m}=0.3175, \Omega_{b}=0.049, h=0.6711, n_{s}=0.9624, \sigma_{8}=0.834\right.$, $\left.M_{\nu}=0\right)$ and HOD parameters $\left(\log M_{\min }=13.65, \sigma_{\log M}=0.2, \log M_{0}=14.0, \alpha=1.1, \log M_{1}=\right.$ 14.0), which are based on the best-fit HOD parameters for the SDSS $M_{r}<21.5$ and -22 samples $\frac{16}{\text {. }}$ They are designed for accurately estimating the covariance matrices of high dimensional galaxy clustering observables. It also includes 60,000 galaxy catalogs that are constructed at 24 different parameter values and designed for accurately estimating the derivatives with respect to each of the 11 parameters. The parameter values are determined by varying each parameter of the fiducial values one step above and below. In total, the MOLINO suite includes 75, 000 galaxy catalogs.

$\sqrt[1]{\text { changhoonhahn.github.io/molino }}$ 


\section{The reconstruction on the MOLINO mocks}

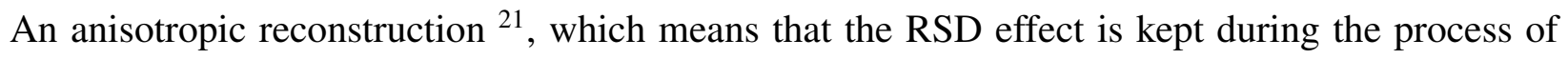
reconstruction, is performed on each realisation of the MOLINO galaxy mocks with two choices of the smoothing scale, $S=10$ and $20 h^{-1} \mathrm{Mpc}$ (All results presented in the main text are for the $10 h^{-1}$ Mpc case, while results for $20 h^{-1} \mathrm{Mpc}$ are shown in the Supplement information). Specifically, a smoothing is performed by convolving density field with the kernel $K(k)=\exp \left[-(k S)^{2} / 2\right]$ in Fourier space. Then the displacement vector is solved using the Zeldovich approximation, i.e., $\tilde{\mathbf{s}}(\mathbf{k})=-\frac{i \mathbf{k}}{k^{2}} \frac{\delta(\mathbf{k})}{b+f \mu^{2}} K(k)$, where $\delta$ denotes the nonlinear redshift-space overdensity, $b$ is the linear bias measured from the mocks, and $f$ is the fiducial logarithmic growth rate for the MOLINO mocks. An inverse Fourier transformation on $\tilde{\mathbf{s}}$ returns the configuration-space displacement field $\mathbf{s}(\mathbf{x})$, which is used to move both the galaxies and randoms by $\mathbf{s}(\mathbf{x})+f(\mathbf{s} \cdot \hat{\mathbf{z}}) \hat{\mathbf{z}}$.

\section{Measurement of the power spectrum multipoles}

The multipoles of both the pre- and post-reconstructed density fields are measured using an FFT-based estimator $^{1}$ that is implemented in $\mathrm{N}$-body $\mathrm{kit}^{2}$. The shot-noise, which reflects the discreteness of the density field, is removed as a constant for the monopole of the auto-power.

Care needs to be taken when measuring the cross-power spectrum between the pre- and post-reconstructed density fields, since the raw measurement using the FFT-based estimator is contaminated by a scale-dependent shot-noise: on large scales, the post-reconstructed field resembles the unreconstructed one, making the cross-power spectrum essentially an auto-power, thus it is subject to a shot-noise component. On small scales, however, the shot-noise largely drops because 
the two fields effectively decorrelate.

To obtain a shot-noise-free cross-power spectrum, we adopt the "half-sum (HS) half-difference (HD)" approach, motivated by that used for measuring the shot-noise-free auto-power spectrum ${ }^{3}$. We start by randomly dividing the catalog into two halves, dubbed $\delta_{1}$ and $\delta_{2}$, and the corresponding reconstructed density fields are $R_{1}$ and $R_{2}$, respectively.

Let

$$
\mathrm{HS} \equiv \frac{\delta_{1}+\delta_{2}}{2}, \mathrm{HD} \equiv \frac{\delta_{1}-\delta_{2}}{2},
$$

and

$$
\mathrm{HS}^{\mathrm{R}} \equiv \frac{\mathrm{R}_{1}+\mathrm{R}_{2}}{2}, \mathrm{HD}^{\mathrm{R}} \equiv \frac{\mathrm{R}_{1}-\mathrm{R}_{2}}{2} .
$$

Then $\mathrm{HS}^{(\mathrm{R})}$ contains both the signal and noise, but $\mathrm{HD}^{(\mathrm{R})}$ only contains noise. Hence the noise-free cross-power spectrum can be estimated by,

$$
P_{\text {cross }}=\left\langle\mathrm{HS}, \mathrm{HS}^{\mathrm{R}}\right\rangle-\left\langle\mathrm{HD}, \mathrm{HD}^{\mathrm{R}}\right\rangle=\frac{\left\langle\delta_{1}, \mathrm{R}_{2}\right\rangle+\left\langle\delta_{2}, \mathrm{R}_{1}\right\rangle}{2} .
$$

This allows for an estimation of the $k$-dependent shot noise of the cross power spectrum, as a by-product. As shown in Supplement Figures 7 and 8 (for cases with $S=10$ and $20 h^{-1} \mathrm{Mpc}$, respectively), the noise is anisotropic thus it exists even for multipoles with $\ell \neq 0$, which could be understood from statistics and perturbation theory ${ }^{4}$. 


\section{Measurement of the bispectrum monopole}

We measure the galaxy bispectrum monopole, $B_{0}$, for all of the MoLINO catalogs using the publicly available pySpectrum package $e^{2}$ Galaxy positions are first interpolated onto a grid with $N_{\text {grid }}=360$ using a fourth-order interpolation scheme and then Fourier transformed to obtain $\delta(k)$. Afterwards $B_{0}$ is estimated using

$$
B_{0}\left(k_{1}, k_{2}, k_{3}\right)=\frac{1}{V_{B}} \int_{k_{1}} \mathrm{~d}^{3} q_{1} \int_{k_{2}} \mathrm{~d}^{3} q_{2} \int_{k_{3}} \mathrm{~d}^{3} q_{3} \delta_{\mathrm{D}}\left(\mathbf{q}_{\mathbf{1 2 3}}\right) \delta\left(\mathbf{q}_{\mathbf{1}}\right) \delta\left(\mathbf{q}_{\mathbf{2}}\right) \delta\left(\mathbf{q}_{\mathbf{3}}\right)-B_{0}^{\mathrm{SN}}
$$

where $\delta_{D}$ is the Dirac delta function, $V_{B}$ is the normalization factor proportional to the number of triplets that can be found in the $k_{1}, k_{2}, k_{3}$ triangle bin, and $B_{0}^{\mathrm{SN}}$ is the Poisson shot noise correction term. Triangle configurations are defined by $k_{1}, k_{2}, k_{3}$ bins of width $\Delta k=3 k_{f}$, where $k_{f}=$ $2 \pi /\left(1000 h^{-1} \mathrm{Mpc}\right)$.

Data Availability The data that support the plots within this paper and other findings of this study are available from the corresponding author upon reasonable request.

1. Hand, N., Li, Y., Slepian, Z. \& Seljak, U. An optimal FFT-based anisotropic power spectrum estimator. JCAP 2017, 002 (2017). 1704 . 02357.

2. Hand, N. et al. nbodykit: An Open-source, Massively Parallel Toolkit for Large-scale Structure. Astron. J. 156, 160 (2018). 1712.05834.

3. Ando, S., Benoit-Lévy, A. \& Komatsu, E. Angular power spectrum of galaxies in the 2MASS Redshift Survey. Mon. Not. Roy. Astron. Soc. 473, 4318-4325 (2018). 1706.05422.

\footnotetext{
2 https://github.com/changhoonhahn/pyspectrum
} 
4. Zhao, R. et al. (To appear). 


\section{Supplementary Information}

\section{An independent mock test for demonstration}

To support the main text, here we present an independent mock test using a suite of high-resolution dark matter-only mocks, to forecast the sensitivity of the BAO and RSD parameters. The mock is a suite of $4000 N$-body simulations with $512^{3}$ dark matter particles in a $L=500 h^{-1} \mathrm{Mpc}$ box at $z=1.02$. We perform the anisotropic Lagrangian reconstruction ${ }^{1}$ on each realisation of the mocks with a smoothing scale $S=10 h^{-1} \mathrm{Mpc}$, before measuring the multipoles (up to $\ell=4$ ) of $P_{\text {pre }}, P_{\text {post }}$ and $P_{\text {cross }}$, respectively. To investigate the sensitivity on cosmological parameters, we perform a Fisher matrix analysis ${ }^{14}$ on BAO parameters $\left(\alpha_{\perp}, \alpha_{\|}\right)$and a parameter describing the change of velocities along the line of sight $(\Delta v)$ by computing the derivatives with respect to each parameter numerically. The parameter $\Delta v$ mimics the change of the linear growth rate on large scales, but it also changes the velocity of particles coherently on small scales.

The result is shown in Supplement Figure 1. From panel a we see that the amplitude of $P_{\text {cross }}$ decreases dramatically with scales, indicating a decorrelation effect between $P_{\text {pre }}$ and $P_{\text {post }}$ below quasi-nonlinear scales, which is confirmed by the correlation coefficient (the normalised covariance) plotted in panel b. This decorrelation, which is not caused by the shot noise given the noise level in the mocks, is a clear evidence of the complementarity among the power spectra.

The projection onto BAO parameters and $\Delta v$, shown in panels $\mathbf{c}_{1}-\mathbf{c}_{8}$, well demonstrates the advantage for performing a joint analysis using $P_{\text {pre }}, P_{\text {post }}$ and $P_{\text {cross }}$. On large scales, $P_{\text {pre }}$ and 
$P_{\text {post }}$ are both determined by the linear density field, making the power spectra highly correlated, as shown in panel $\mathbf{b}$. As shown in panels $\mathbf{c}_{5}$, the contours derived from $P_{\text {pre }}$ and $P_{\text {post }}$ have similar orientations and we do not gain by combining them. For $k>0.15 h \mathrm{Mpc}^{-1}$, the correlation between $P_{\text {pre }}$ and $P_{\text {post }}$ decreases as the pre-reconstructed density field is dominated by the non-linear field while $P_{\text {post }}$ still retains the correlation with the linear density field. The contours shown in lines in panel $\mathbf{c}_{7}$, which are derived from power spectra in the $k$ range of $[0.2,0.25] h \mathrm{Mpc}^{-1}$, are almost orthogonal to each other, making the constraint from the combined spectra, as illustrated in the shaded region, significantly tightened. On smaller scales, the post-reconstructed density field is also dominated by the non-linear field and the orientations of the contours are again aligned and the complementarity on smaller scales weakens. This shows that the level of nonlinearity in the power spectrum determines the degeneracies between parameters. Since $P_{\text {pre }}$ and $P_{\text {post }}$ are affected by different levels of nonlinearities on a given scale, which gives rise to different degeneracies, a joint analysis using both $P_{\text {pre }}$ and $P_{\text {post }}$ (and $P_{\text {cross }}$ ) can yield a better constraint by breaking the degeneracies. 

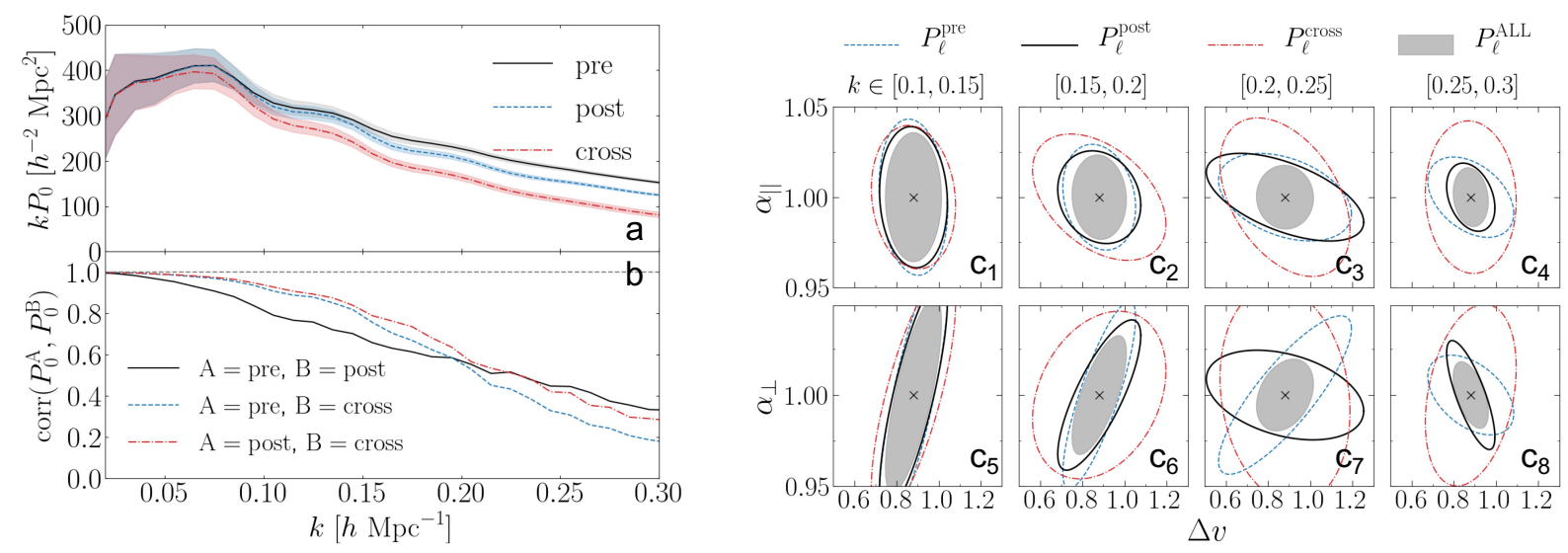

Supplement Figure 1. An illustration of the complementarity between $P_{\text {pre }}, P_{\text {post }}$ and $P_{\text {cross }}$ (with the smoothing scale $S=10 h^{-1} \mathrm{Mpc}$ ) when performing the reconstruction on the 4000

\section{N-body mocks.}

Panel a: the mean (lines) and $68 \%$ uncertainty (shades) of the measured three types of power spectrum monopole (multiplied by the wavenumber $k$ ); Panel b: the measured correlation coefficient between various types of power spectrum monopoles. The dashed horizontal line shows a prefect correlation (i.e., corr $=1$ ) for a reference; Panels c: the $68 \% \mathrm{CL}$ contour plots for $\mathrm{BAO}$ and RSD parameters including $\alpha_{\|}, \alpha_{\perp}$ and $\Delta v$ derived from three types of power spectrum multipoles $(\ell=0,2,4)$ separately (lines) and jointly (filled). Power spectra used in different $k$ ranges are shown in different columns, as illustrated in the legend. 


\section{Supplementary figures for analyses using the Molino mocks}

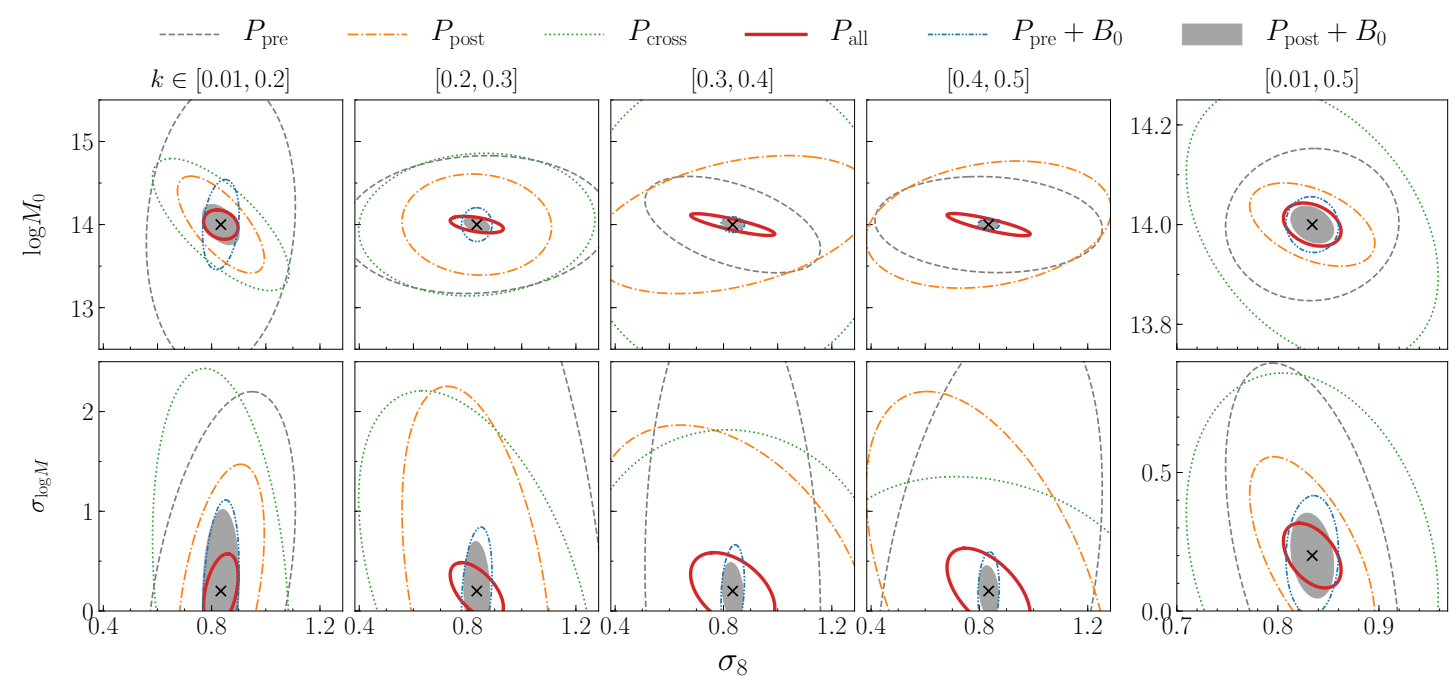

Supplement Figure 2. The 68\% CL contour plots on $\left(\sigma_{8}, \log M_{0}\right)$ and $\left(\sigma_{8}, \sigma_{\log M}\right)$ for different combination of summary statistics in several $k$ ranges. 


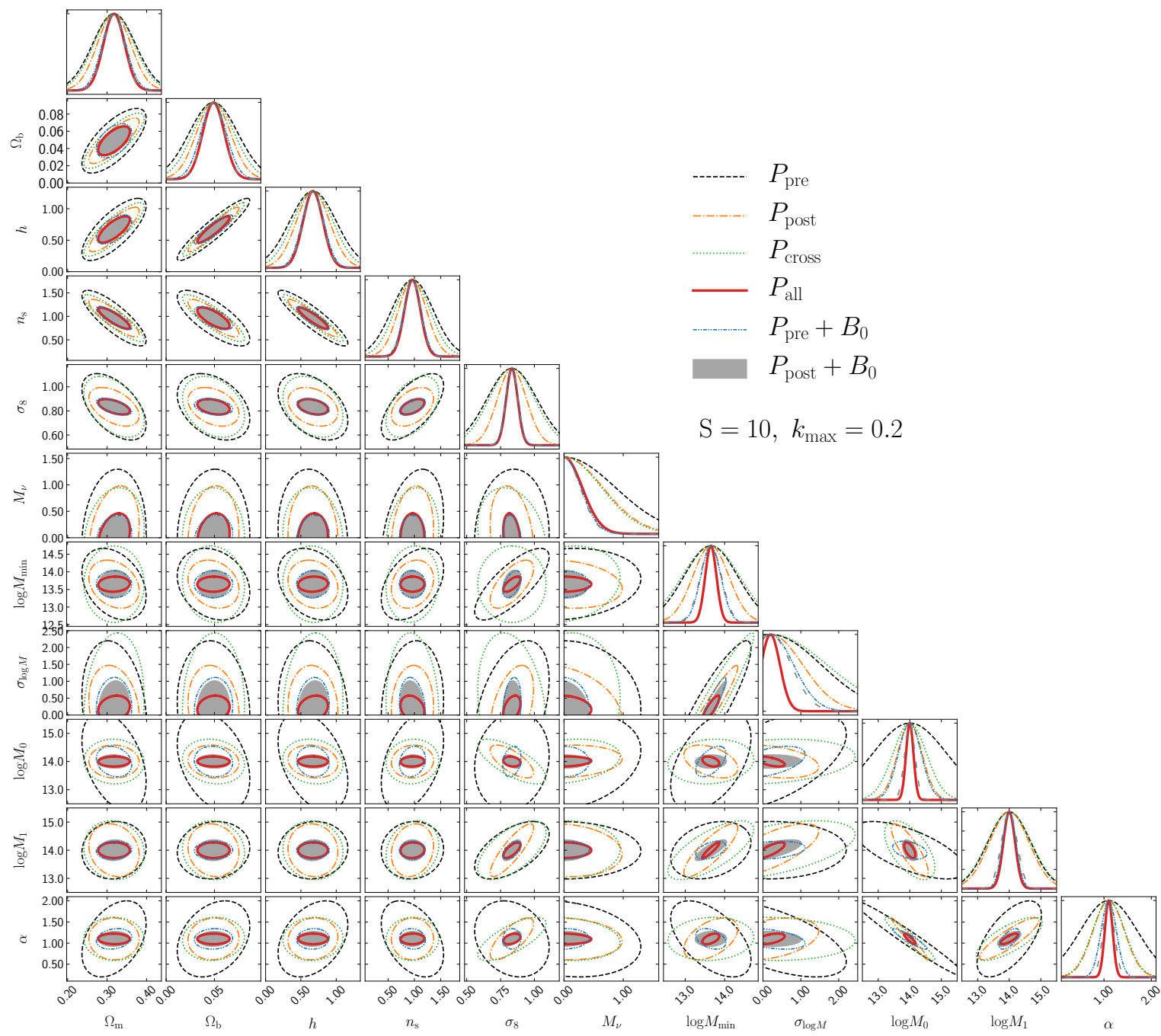

Supplement Figure 3. The 1D posterior distribution and $68 \% \mathrm{CL}$ contour plots for cosmological and HOD parameters up to $k_{\max }=0.2 h \mathrm{Mpc}^{-1}$. The smoothing scale for the reconstruction is $S=10 h^{-1} \mathrm{Mpc}$. 


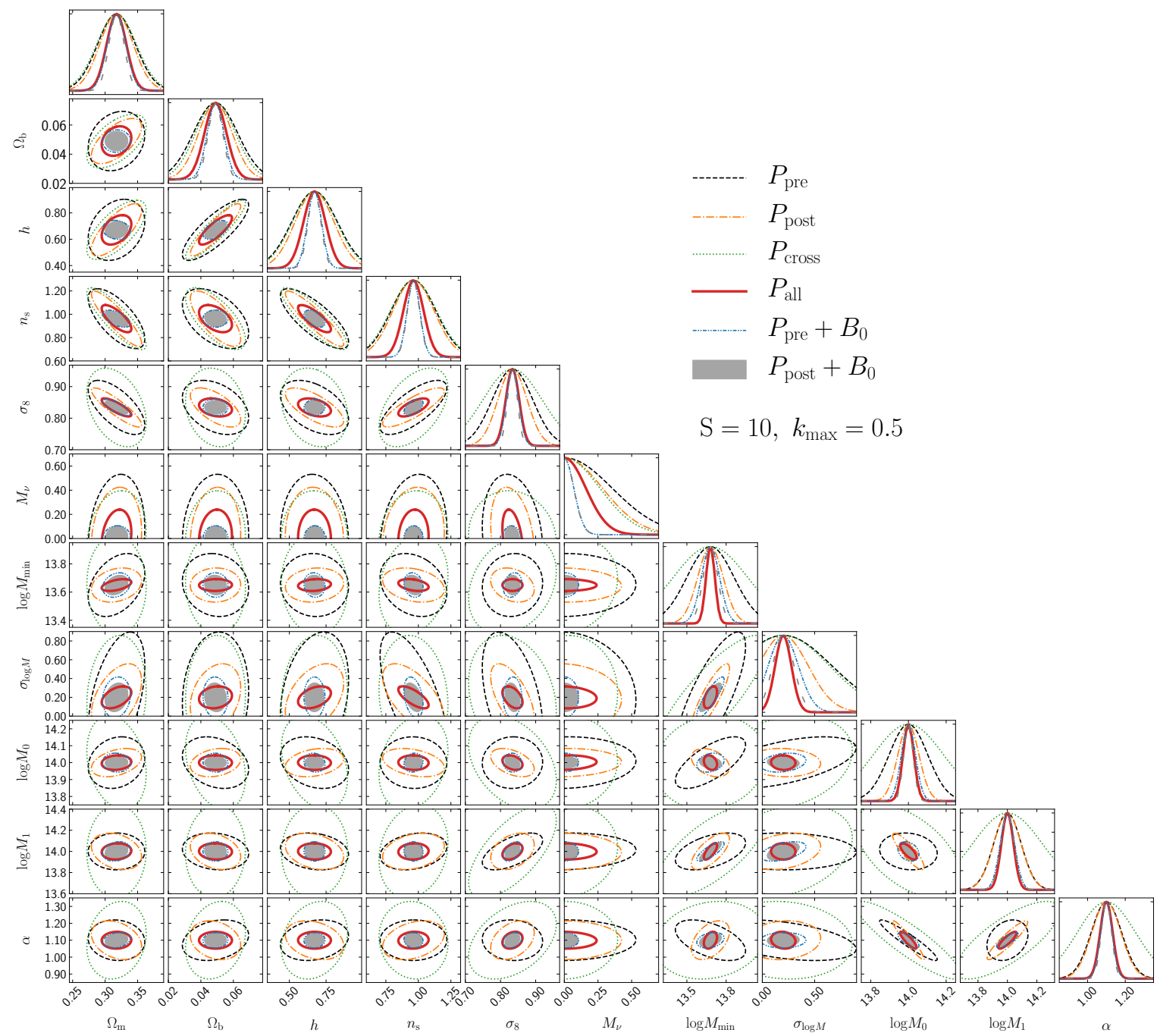

Supplement Figure 4. The 1D posterior distribution and $68 \%$ CL contour plots for cosmological and HOD parameters up to $k_{\max }=0.5 h \mathrm{Mpc}^{-1}$. The smoothing scale for the reconstruction is $S=10 h^{-1} \mathrm{Mpc}$. 


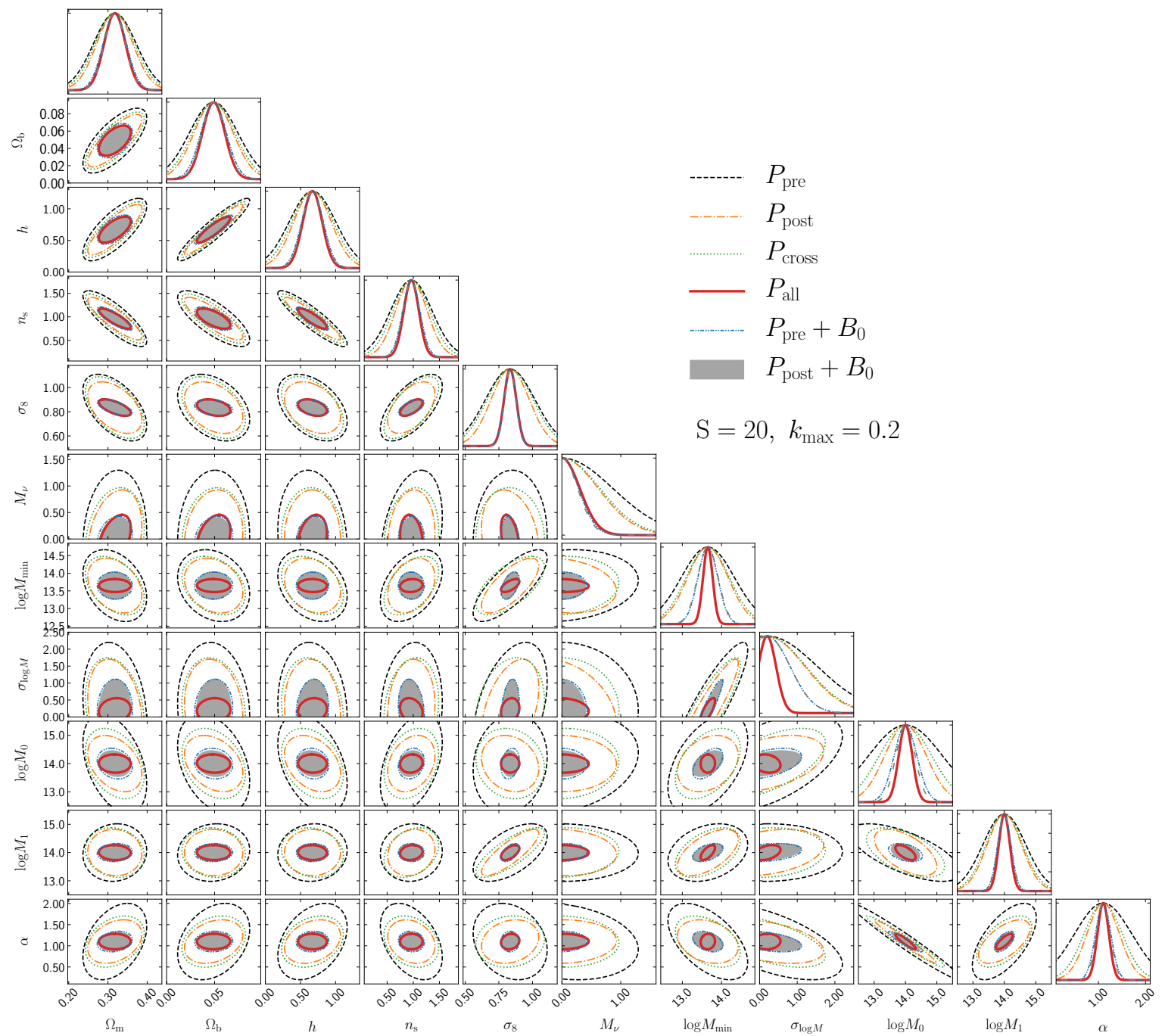

Supplement Figure 5. The 1D posterior distribution and $68 \%$ CL contour plots for cosmological and HOD parameters up to $k_{\max }=0.2 h \mathrm{Mpc}^{-1}$. The smoothing scale for the reconstruction is $S=20 h^{-1} \mathrm{Mpc}$. 


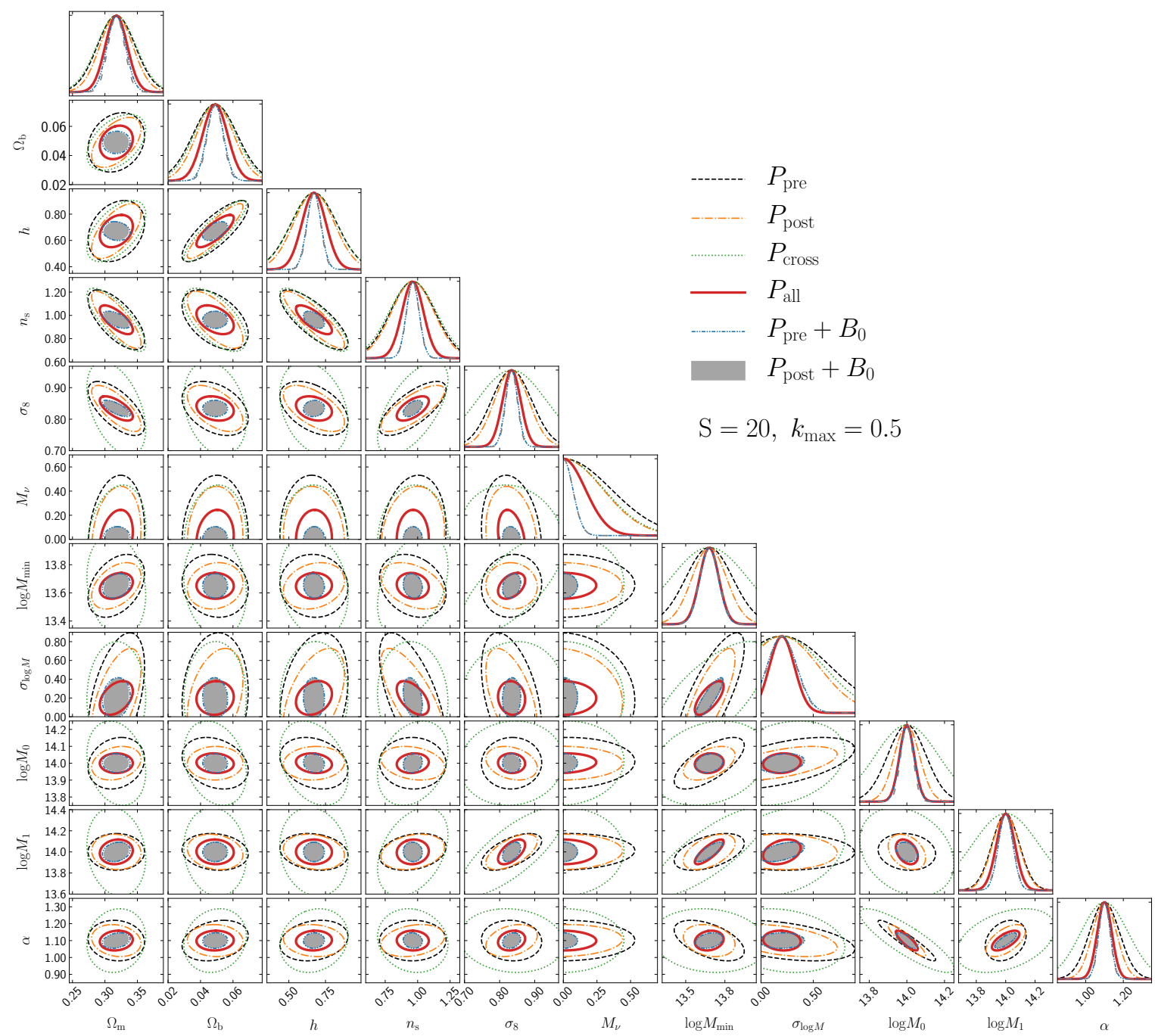

Supplement Figure 6. The 1D posterior distribution and $68 \%$ CL contour plots for cosmological and HOD parameters up to $k_{\max }=0.5 h \mathrm{Mpc}^{-1}$. The smoothing scale for the reconstruction is $S=20 h^{-1}$ Mpc. 


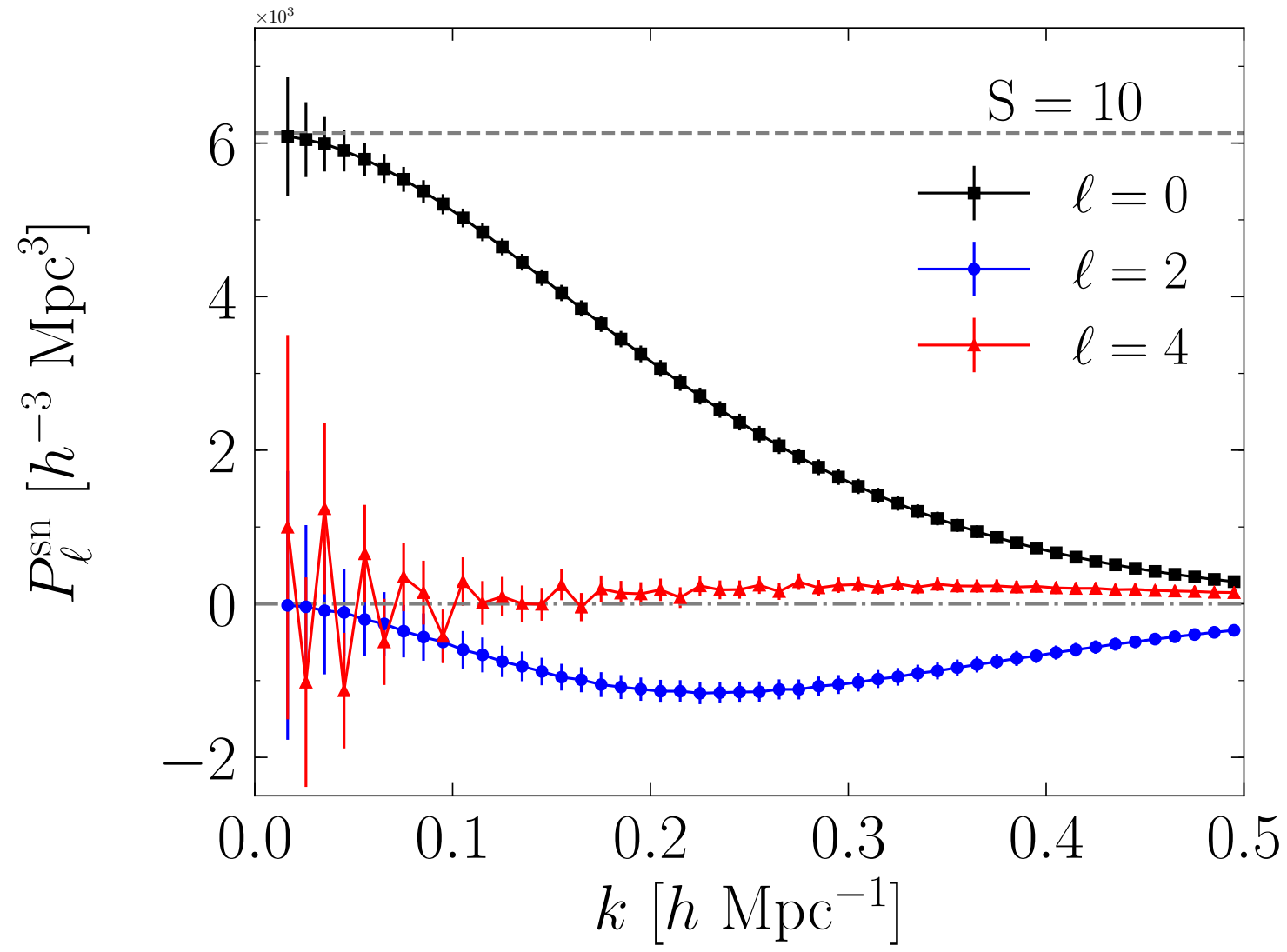

Supplement Figure 7. The measured shot-noise for the cross power spectrum multipoles. The smoothing scale $S=10 h^{-1} \mathrm{Mpc}$ is used for the reconstruction. 


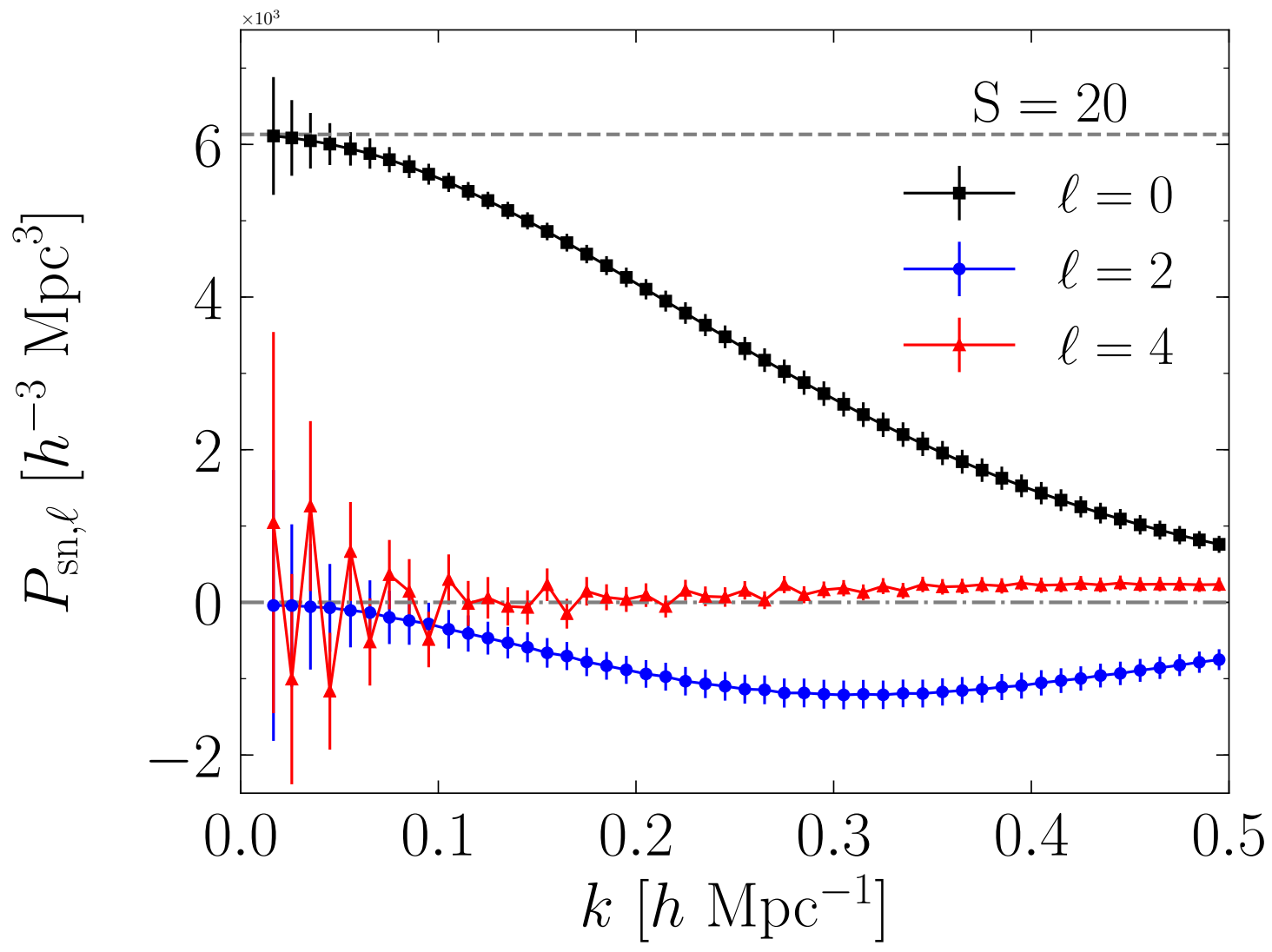

Supplement Figure 8. The measured shot-noise for the cross power spectrum multipoles. The smoothing scale $S=20 h^{-1} \mathrm{Mpc}$ is used for the reconstruction.

1. Seo, H.-J., Beutler, F., Ross, A. J. \& Saito, S. Modeling the reconstructed BAO in Fourier space. Mon. Not. Roy. Astron. Soc. 460, 2453-2471 (2016). 